\section{Disparités d'adoption des technologies en pédagogie universitaire : un aperçu empirique}

Challenges Impeding Technology Adoption in Higher Education: An Empirical View
Simon COLLIN

Université du Québec à Montréal collin.simon@uqam.ca

Glorya PELLERIN Université du Québec en Abitibi-Témiscamingue glorya.pellerin@uqat.ca

André BLANCHARD

Université du Québec en Abitibi-Témiscamingue andre.blanchard@uqat.ca

Benoît CORDELIER

Université du Québec à Montréal cordelier.benoit@uqam.ca

Hamid SAFFARI

Université du Québec à Montréal saffari.hamid@uqam.ca

Recherche scientifique avec données empiriques

\section{Résumé}

Bien que les études traitant de l'intégration des technologies en pédagogie universitaire soient relativement nombreuses, peu d'entre elles se sont penchées sur les disparités d'adoption des technologies parmi les enseignants universitaires. Aussi, l'objectif de cet article est de caractériser les profils d'enseignants universitaires adoptant les technologies. Un questionnaire a été rempli par 391 enseignants de deux universités du Québec. Des analyses par clusters permettent d'identifier trois profils d'enseignants universitaires intégrant les technologies relativement à trois volets de leur enseignement : préparation et gestion, pilotage, et développement professionnel. Les résultats laissent penser que les disparités d'adoption ne se sont pas résorbées malgré la succession de plus en plus rapide des technologies en pédagogie universitaire.

\section{Mots-clés}

Pédagogie universitaire, adoption des technologies, enseignants universitaires

\begin{abstract}
Although a considerable number of studies have been conducted on technologies and higher education, few of them have addressed the disparity of technology adoption among university teachers. The objective of this study is to characterize the profiles of university teachers adopting technologies. 391 university teachers from two universities in Quebec completed a questionnaire. The cluster analyses made it possible to identify three profiles of university teachers adopting technologies in relationship to three aspects of their teaching: preparation and delivery of teaching/learning activities; instructional management; professional development. Overall, the results indicate that disparity of technology adoption among university teachers has not improved despite an increasingly rapid succession of technologies in higher education.
\end{abstract}

\section{Keywords}

Keywords: higher education, technology adoption, university teachers 
La quête de l'efficacité des technologies en pédagogie universitaire

En tant que domaine de recherche, la pédagogie universitaire a consacré une place de choix à l'intégration des technologies (Albero, 2011; De Ketele, 2010). Sur le plan institutionnel et pédagogique, des efforts financiers et humains massifs ont été consentis (Edmunds, Thorpe et Conole, 2012; Geoghegan, 1994) sur l'idée d'augmenter la qualité de l'enseignement et de l'apprentissage universitaire et d'en démocratiser l'accès (Gul, Arif et Yousaf, 2013). La communauté scientifique y a pleinement contribué en s'attelant massivement à révéler l'efficacité des technologies par l'étude de leurs impacts sur la situation d'enseignement et d'apprentissage (Kerr, 1996). Ce faisant, les technologies en pédagogie universitaire, et plus largement en éducation sont « approached as an inherently 'positive project' » (Selwyn, 2015, p. 251). Cette tendance est particulièrement saillante à chaque apparition d'une "nouvelle» technologie, laquelle fait l'objet de discours en grande partie « jovialistes » sur son potentiel présumé à modifier, pour le mieux, la pédagogie universitaire (Collin et Karsenti, 2013). Le dernier exemple à ce jour est le cas des Massive Open Online Course (MOOC), qui a - aussi soudainement que fortement - accaparé l'espace public, médiatique et scientifique simplement parce qu'il s'agissait de la toute dernière innovation technopédagogique. Il est possible de penser que cette entrée par l'outil est nuisible dans la mesure où elle met les motivations scientifiques au diapason de la diffusion technologique. Or, comme les technologies se succèdent de plus en plus rapidement en pédagogie universitaire, les chercheurs disposent de moins en moins de temps pour élaborer une base de connaissances sur chacune d'elles avant de s'affairer à étudier la suivante. S'ensuit une fragmentation de l'avancement des connaissances, où chaque technologie fait l'objet de recherches partielles à son arrivée, et en relative autonomie des précédentes. Cet état de fait a été régulièrement rapporté dans la littérature scientifique du domaine (Albero, 2011; Collin et Karsenti, 2013; Baron et Bruillard, 1996; Cuban, 1986).

\section{Les disparités d'adoption des technologies par les enseignants universitaires}

Rythmées par l'apparition successive de nouvelles technologies, les études sur les technologies en pédagogie universitaire tendent à se concentrer sur les premiers temps d'adoption des technologies par les enseignants universitaires (Kirkup, Sizmur, Sturman et Lewis, 2005) et à négliger la suite de son adoption par le reste du corps enseignant.

Pourtant, certains modèles, comme celui des innovations technologiques de Rogers (2003), bien qu'il ne soit pas issu de la pédagogie universitaire, ouvrent des perspectives intéressantes pour éclairer le processus d'adoption des technologies par les enseignants universitaires à moyen et à long terme. L'adoption est définie par Rogers (2003) comme le fait, pour un individu, de décider du « full use of an innovation as the best course of action available » (p. 177). Dans le cadre de cet article, les termes « utilisation » et « intégration » seront employés comme synonymes de celui d'adoption. Le modèle de Rogers (2003) présente l'adoption d'une innovation, technologique ou sociale, comme un processus communicationnel inscrit dans le temps et rendant compte du taux d'adoption dans un ensemble social observé. C'est donc une vision quantitative qui est favorisée, se concentrant sur le rythme de l'acceptation ou du refus de l'innovation. Graphiquement, cela se traduit par une courbe en S comme l'avait pressenti De Tarde dans son ouvrage Les lois de l'imitation (1890). L'adoption de l'innovation démarre d'un groupe restreint vers l'ensemble de la population. Rogers (2003) propose alors une catégorisation qui distingue cinq profils : 1) les pionniers (innovators), qui regroupent les utilisateurs intéressés par les innovations technologiques pour elles-mêmes, indépendamment de ce qu'elles peuvent leur apporter; 2) les adopteurs précoces (early adopters), qui explorent le potentiel des technologies à améliorer, voire à révolutionner, une situation; 3) la majorité précoce, qui adopte les technologies une fois qu'elle entrevoit concrètement quels en sont les apports; 4) la majorité tardive, qui se distingue de la majorité précoce en termes de degré : 
disposant de moins de compétences technologiques, elle a besoin de plus de temps et d'arguments pour juger de l'intérêt d'adopter une technologie; 5) et les réfractaires, qui sont peu susceptibles d'adopter une technologie, car ils y portent peu d'intérêt. Bien qu'il ne soit pas sans critiques, notamment pour sa vision mécaniste et uniquement quantitative de la diffusion de l'innovation, ce modèle a néanmoins permis d'apporter un éclairage plus précis et différencié sur le processus d'adoption des technologies au sein d'une population (voir Millerand, 1998).

Geoghegan (1994), en appliquant les travaux de Rogers (2003) et de Moore (1991) à la pédagogie universitaire, offre un bon aperçu du potentiel de ce modèle. D'après lui (1994), un problème fondamental de l'intégration des technologies à l'université réside dans le fait qu'une innovation technologique donnée parvient rarement à être diffusée au-delà des deux premiers profils d'utilisateurs, les pionniers et les adopteurs précoces. Regroupant environ $15 \%$ de la population enseignante universitaire, ce profil minoritaire correspond à des caractéristiques sociales et psychologiques particulières : il s'agit d'enseignants qui, entre autres, ont un fort intérêt préalable pour les technologies, qui ne sont pas échaudés par la prise de risques, et qui sont relativement autonomes sur le plan technologique et pédagogique (Geoghegan, 1994). Se crée alors un fossé entre une minorité d'enseignants « innovants » et une majorité d'enseignants pour qui l'intégration des technologies nécessiterait plus de temps et de soutien que ce que l'institution permet pour être durable et signifiante. Ainsi, le constat principal de Geoghegan (1994) est celui d'une disparité d'adoption des technologies au sein des enseignants universitaires. Des recherches effectuées sur les facteurs d'adoption des technologies par les enseignants universitaires (Ben Youssef, Ben Youssef et Dahmani, 2013; Usluel, Aşkar et Baş, 2008)

laissent également entrevoir des degrés d'adoption variables par les enseignants universitaires, bien qu'elles ne renseignent pas sur les disparités en tant que telles.
Depuis les constats de Geoghegan (1994), les technologies se sont succédé à un rythme de plus en plus rapide en pédagogie universitaire, mais peu d'études se sont penchées sur les disparités d'adoption au sein des enseignants universitaires (Kirkup et al., 2005; Selwyn, 2007). Il nous apparaît que le constat de Larose, Lenoir, Karsenti et Grenon (2002) reste encore de mise : « il n’y a actuellement que très peu d'études disponibles qui présentent des données stables au regard des profils d'utilisation des TICE chez les professeurs d'université » (p. 28). Les disparités d'adoption décrites par Geoghegan en 1994 sont-elles encore d'actualité ou se sont-elles résorbées? La pédagogie universitaire s'est-elle appropriée massivement les technologies depuis? Ces questions peuvent sembler anodines; elles n'ont demeurent pas moins essentielles. En effet, il nous apparaît que la question de l'efficacité des technologies en pédagogie universitaire ne peut être dissociée de celle de son adoption massive et durable par le corps enseignant. Car pour que les technologies contribuent de manière signifiante à améliorer la pédagogie universitaire, elles doivent vraisemblablement répondre à deux conditions : 1) d'une part, s'avérer efficaces pour la situation d'enseignement et d'apprentissage, ce qui est au centre des préoccupations scientifiques (voir section La quête de l'efficacité des technologies en pédagogie universitaire); 2) d'autre part, être massivement adoptées par les enseignants universitaires d'une communauté universitaire donnée, ce qui fait l'objet de beaucoup moins d'études. Autrement dit, une technologie, même si elle est jugée efficace pour l'enseignement et l'apprentissage universitaire, voit sa pertinence pédagogique réduite si elle n'est, au final, adoptée que par une poignée d'enseignants. Or, l'absence de résultats récents sur les disparités d'adoption des technologies en pédagogie universitaire ne permet pas de se prononcer sur ce deuxième point. C'est donc dans le souci de fournir un aperçu empirique actuel, bien que partiel, de l'état des disparités d'adoption des technologies par les enseignants universitaires que nous avons mené cette étude. 


\section{Objectif}

La section précédente nous a permis de constater que peu d'études se sont penchées sur les disparités d'adoption des technologies parmi les enseignants universitaires. En nous inspirant des perspectives ouvertes par les travaux sur la diffusion des innovations technologiques et de leur application dans le domaine des technologies en pédagogique universitaire par Geoghegan (1994), l'objectif du présent article est de caractériser les profils d'enseignants universitaires adoptant les technologies. Ce faisant, nous n'avons pas la prétention d'explorer de nouveaux objets de recherche, mais plutôt d'en actualiser certains, pour lesquels il n'existe pas de portrait récent alors qu'ils nous semblent fondamentaux pour apprécier l'évolution et la pertinence des technologies en pédagogie universitaire.

\section{Méthodologie}

Cet article s'inscrit dans le cadre d'une recherche plus large portant sur l'intégration des technologies en pédagogie universitaire, menée par une équipe de chercheurs en provenance de deux universités québécoises : l'une est une université populeuse située en milieu urbain et l'autre est une université de petite taille située en région. Il s'agit donc de deux terrains académiques contrastés, l'idée n'étant pas de les comparer, mais de prendre en compte la diversité des réalités universitaires du Québec. Les participants sont les professeurs réguliers $(52,17 \%)$, les professeurs invités $(2,17 \%)$ et les chargés de cours $(41,3 \%)$ des deux universités, pour un total de 432 répondants. Leur expérience d'enseignement est variable, allant de moins d'un an $(6,05 \%)$ à un à cinq ans $(29,78 \%)$, six à dix ans $(20,58 \%)$ et plus de dix ans $(43,05 \%)$.

\section{Collecte de données}

Pour opérationnaliser, sur le plan méthodologique, l'utilisation des technologies en pédagogie universitaire, nous avons opté pour le référentiel de compétences des futurs enseignants du Québec (Ministère de l'Éducation du Québec, 2001), à défaut de disposer d'un référentiel spécifiquement dédié à l'enseignement universitaire, et à l'instar de plusieurs autres études portant sur l'intégration pédagogique des technologies au Québec (p. ex., Karsenti, Raby, Villeneuve et Gauthier, 2007; Raby, Boegner-Pagé, Charron, Gagnon et Bouchard, 2013; Stockless et Beaupré, 2014; Villeneuve, Karsenti, Raby et Meunier, 2011). Le questionnaire utilisé se composait d'une section socioprofessionnelle et de sections correspondant aux différents volets de l'intégration des technologies en enseignement. En effet, la compétence professionnelle 8 du référentiel indique que les futurs enseignants doivent être formés à utiliser les technologies pour contribuer à la préparation et à la gestion de leur enseignement, au pilotage d'activités pédagogiques et à leur développement professionnel. Pour chacune de ces sections, le questionnaire consistait à cocher dans des listes d'items les technologies utilisées (24 items; p. ex., «Logiciels de cartes conceptuelles (ex. Inspiration, CMapTools) et les usages effectués avec ces technologies (11 items; p. ex., « Recherche d'information »). Les listes d'items ont été générées par une revue de la littérature sur l'intégration des technologies en pédagogie universitaire, selon la méthode proposée par Gall, Gall et Borg (2005).

\section{Analyse des données}

Pour analyser les profils d'adoption des technologies en pédagogie universitaire, nous avons commencé par générer des variables composites à partir de celles relatives aux technologies et aux usages technologiques rapportés par les participants, de façon à obtenir le nombre de technologies et le nombre d'usages technologiques mobilisés par chacun d'eux pour chaque volet d'enseignement. À titre d'exemple, un participant ayant coché six technologies et quatre usages technologiques dans le volet «préparation et gestion de l'enseignement» obtient un score de 6 et de 4 , indépendamment des technologies et des usages technologiques spécifiques qu'il a cochés. Ce type de variables, bien qu'il représente une mesure partielle de l'adoption des technologies, s'avère fiable pour en étudier les 
variations (dans notre cas, les disparités), comme en atteste son utilisation par plusieurs chercheurs (voir, par exemple, Hargittai, 2010; Helsper et Eynon, 2010; Livingstone et Helsper, 2007; Wei, 2012). Il présente notamment l'avantage de faire ressortir les grandes tendances de l'adoption des technologies par les participants, avant de spécifier les résultats à des technologies ou des usages technologiques particuliers. À partir de ces variables génériques, nous avons effectué une analyse par cluster hiérarchique sur les 299 participants n'ayant aucune réponse manquante. Une solution à trois clusters s'est avérée la plus productive, avec laquelle nous avons opéré une ANOVA sur chacune des six variables composites, soit le nombre de technologies et d'usages technologiques rapportés pour la préparation et la gestion de l'enseignement, pour le pilotage d'activités d'enseignement et pour le développement professionnel. Les comparaisons post-hoc ont confirmé que ces trois clusters étaient significativement distincts pour les six variables génériques utilisées.

\section{Résultats}

Les analyses par cluster ont permis de dégager trois profils distincts d'enseignants intégrant les technologies sur la base du plus ou moins grand nombre de technologies et d'usages technologiques qu'ils rapportent intégrer à leur enseignement. Il est ainsi possible de distinguer les petits $(n=92$, soit $30,8 \%$ ), moyens $(n=181$, soit $60,5 \%$ ) et grands utilisateurs $(n=26$, soit $8,7 \%)$. Pour chaque volet d'intégration des technologies en enseignement (préparation et gestion de l'enseignement; pilotage d'activités pédagogiques; développement professionnel), nous présentons de manière descriptive les technologies et les usages technologiques que les participants mobilisent. Dans un souci de concision, nous nous contentons de rapporter les technologies et les usages partagés par l'ensemble des participants, avant d'en tirer quelques remarques sur leur distribution au sein des trois profils d'enseignants universitaires.

\section{Planification et gestion de l'enseignement}

L'analyse par cluster révèle que les petits, moyens et grands utilisateurs mobilisent en moyenne 6,8 et 13 technologies pour la planification et la gestion de leur enseignement. Sur la base de ces chiffres, nous avons sélectionné les 6,8 et 13 technologies qui sont les plus fréquemment rapportées par les petits, moyens, et grands utilisateurs. Le tableau 1 résulte de ce croisement du nombre moyen de technologies par profil d'utilisateur avec les technologies les plus populaires indiquées par chacun d'eux. La colonne de gauche du tableau liste par ordre décroissant les 13 technologies les plus fréquemment rapportées par les participants. On voit par exemple que les logiciels de traitement de texte sont la technologie la plus populaire, suivie des logiciels de présentation et du courrier électronique. Les trois colonnes de droite indiquent les pourcentages de fréquence pour chaque profil d'utilisateur. Ainsi, le logiciel de traitement de texte est rapporté par 93,50\%, $98,90 \%$ et $100 \%$ des petits, moyens et grands utilisateurs respectivement. Comme les petits utilisateurs mettent à profit un nombre moyen de 6 technologies pour la planification et la gestion de leur enseignement, nous ne présentons que les 6 technologies qu'ils rapportent le plus fréquemment, car ce sont celles qui caractérisent le plus ce volet de leur enseignement. De la même manière, les moyens et les grands utilisateurs rapportent respectivement un nombre moyen de 8 et 13 technologies, de sorte que nous rapportons les 8 et 13 technologies les plus populairement rapportées. Nous pouvons en retenir que les logiciels de traitement de texte (respectivement, 93,5\%, 98,9\% et $100 \%$ des petits, moyens et grands utilisateurs), les logiciels de présentation (respectivement, 90,2 \%, 96,1 \% et 96,2\% des petits, moyens et grands utilisateurs), le courriel (respectivement, 90,6\%, 92,3\% et 80,4\% des petits, moyens et grands utilisateurs), les moteurs de recherche sur Internet (respectivement, 78,3\%, 90,6\% et $96,2 \%$ des petits, moyens et grands utilisateurs) et les plateformes d'enseignement (respectivement, 72,8 \%, $85,6 \%$ et $92,3 \%$ des petits, moyens et grands utilisateurs) sont les technologies les plus fréquemment rapportées par une large majorité des participants de chaque profil. 
Il est intéressant de noter que le courrier électronique est plus utilisé par les petits $(90,6 \%)$ et les moyens $(92,3 \%)$ utilisateurs que par les grands (80,4\%). À l'inverse, ces derniers recourent davantage aux forums de discussion $(65,4 \%)$ et aux réseaux sociaux $(50 \%)$ ce qui laisse penser que leur utilisation plus faible du courriel s'explique par l'utilisation d'une diversité d'outils de communication.

\section{Tableau 1}

Pourcentage de technologies les plus fréquemment utilisées pour le volet de planification et de gestion de l'enseignement en fonction du nombre moyen de technologies rapporté pour chaque profil d'utilisateur

\begin{tabular}{|l|c|c|c|}
\hline $\begin{array}{l}\text { Classement par ordre décroissant des technologies les plus } \\
\text { fréquemment rapportées }\end{array}$ & $\begin{array}{l}\text { Petits utilisateurs } \\
\text { (6 tech. utilisées en } \\
\text { moyenne) }\end{array}$ & $\begin{array}{l}\text { Moyens utilisateurs } \\
\text { (8tech. utilisées en } \\
\text { moyenne) }\end{array}$ & $\begin{array}{l}\text { Grands utilisateurs } \\
\text { (13 tech. utilisées en } \\
\text { moyenne) }\end{array}$ \\
\hline Logiciel de traitement de texte (Word, OpenOffice) & 93,5 & 98,9 & 100 \\
\hline Logiciel de présentation (PowerPoint, Prezi) & 90,2 & 96,1 & 96,2 \\
\hline Courrier électronique (Outlook, Hotmail) & 90,6 & 92,3 & 80,4 \\
\hline Moteur de recherche sur Internet (Google, Yahoo) & 78,3 & 90,6 & 96,2 \\
\hline Plateforme d'enseignement (Moodle, Claroline) & 72,8 & 85,6 & 92,3 \\
\hline Sites Web reliés au contenu de votre cours & 39,1 & 59,1 & 84,6 \\
\hline DVD & & 39,8 & 57,7 \\
\hline Planification de rencontres et sondages (Doodle) & & 37,6 & 61,5 \\
\hline Forum de discussion & & & 65,4 \\
\hline Logiciel d'enregistrement audio-vidéo et de capture d'écran & & & 53,8 \\
\hline (Panopto, Debut) & & & 53,8 \\
\hline Outil de recherche bibliographique (Repère, Copernic) & & & 50 \\
\hline Réseaux sociaux (Twitter, Facebook) & & & 46,2 \\
\hline Wiki & & & \\
\hline
\end{tabular}

Concernant les usages qu'ils font de ces technologies, les petits, moyens et grands utilisateurs ont une moyenne de 5, 8 et 9 usages respectivement comme le montre le tableau 2. 
Tableau 2

Pourcentage des usages technologiques les plus fréquemment mobilisés pour le volet de planification et de gestion de l'enseignement en fonction du nombre moyen d'usages technologiques rapporté pour chaque profil d'utilisateur

\begin{tabular}{|l|c|c|c|}
\hline $\begin{array}{l}\text { Classement par ordre décroissant des usages les plus } \\
\text { fréquemment rapportés }\end{array}$ & $\begin{array}{c}\text { Petits utilisateurs } \\
\text { (5 usages en } \\
\text { moyenne) }\end{array}$ & $\begin{array}{c}\text { Moyens utilisateurs } \\
\text { (8 usages en } \\
\text { moyenne) }\end{array}$ & $\begin{array}{c}\text { Grands utilisateurs } \\
\text { (9 usages en } \\
\text { moyenne) }\end{array}$ \\
\hline Concevoir des cours (déroulement, matériel...) & 91,3 & 97,2 & 100 \\
\hline Élaborer des documents d'animation, de présentation & 80,4 & 95 & 100 \\
\hline Rechercher & 73,9 & 92,8 & 92,3 \\
\hline $\begin{array}{l}\text { Consulter, communiquer et partager (collègues, étudiants, } \\
\text { sites d'informations spécifiques) }\end{array}$ & 59,8 & 93,9 & 100 \\
\hline Évaluer et transmettre des résultats & 59,8 & 87,8 & 92,3 \\
\hline $\begin{array}{l}\text { Prendre des notes ou des informations reliées au contenu } \\
\text { de votre cours (sur un document Word) }\end{array}$ & & 79,6 & 96,2 \\
\hline $\begin{array}{l}\text { Prendre des rendez-vous pour des rencontres avec les } \\
\text { étudiants }\end{array}$ & & 76,8 & 76,9 \\
\hline $\begin{array}{l}\text { Effectuer des rétroactions et supporter les apprentissages } \\
\text { des étudiants }\end{array}$ & & 74,6 & 92,3 \\
\hline $\begin{array}{l}\text { Gérer des documents, des contenus (regroupement ou } \\
\text { classement d'informations) }\end{array}$ & & & 88,5 \\
\hline Compiler (regroupement ou classement d'informations) & & & 76,9 \\
\hline
\end{tabular}

En croisant ces moyennes avec les usages effectifs les plus fréquemment rapportés (colonne de gauche du tableau 2), il apparaît que les usages qui caractérisent le plus les participants sont la conception de cours (respectivement, 91,3\%, 97,2\% et $100 \%$ des petits, moyens et grands utilisateurs), l'élaboration de documents (respectivement, 80,4\%, 95\% et $100 \%$ des petits, moyens et grands utilisateurs), la recherche d'information (respectivement, 73,9\%, 92,8 \% et 92,3\% des petits, moyens et grands utilisateurs), la consultation, la communication et le partage (respectivement, 59,8\%, 93,9\% et $100 \%$ des petits, moyens et grands utilisateurs) ainsi que la gestion des évaluations (respectivement, 59,8\%, 87,8 \% et 92,3\% des petits, moyens et grands utilisateurs).

Il est intéressant de noter que le différentiel le plus élevé entre les petits et grands utilisateurs concerne la consultation, la communication et le partage (respectivement, 59,8\% et $100 \%$ ), ce qui rejoint l'idée énoncée plus haut que les grands utilisateurs se caractérisent notamment par des technologies et usages diversifiés pour communiquer. 


\section{Pilotage d'activités d'enseignement}

Les technologies sont moins utilisées durant le pilotage d'activités d'enseignement que durant la phase de préparation et de gestion, avec une moyenne de 3 (petits utilisateurs), 5 (moyens utilisateurs) et 11 technologies (grands utilisateurs) comme l'indique le tableau 3.

\section{Tableau 3}

Pourcentage de technologies les plus fréquemment utilisées pour le volet de pilotage d'activités d'enseignement en fonction du nombre moyen de technologies rapporté pour chaque profil d'utilisateur

\begin{tabular}{|l|l|c|c|}
\hline $\begin{array}{l}\text { Classement par ordre décroissant des technologies les plus } \\
\text { fréquemment rapportées }\end{array}$ & $\begin{array}{l}\text { Petits utilisateurs } \\
\text { (3 tech. utilisées en } \\
\text { moyenne) }\end{array}$ & $\begin{array}{l}\text { Moyens utilisateurs } \\
\text { (5 tech. utilisées en } \\
\text { moyenne) }\end{array}$ & $\begin{array}{l}\text { Grands utilisateurs } \\
\text { (11 tech. utilisées } \\
\text { en moyenne) }\end{array}$ \\
\hline Logiciel de présentation (PowerPoint, Prezi) & 85,9 & 92,8 & 100 \\
\hline Logiciel de traitement de texte (Word, OpenOffice) & 51,1 & 66,3 & 84,6 \\
\hline Plateforme d'enseignement (Moodle, Claroline) & 41,3 & 63 & 84,6 \\
\hline Moteur de recherche sur Internet (Google, Yahoo) & & 58,6 & 76,9 \\
\hline Sites Web reliés au contenu de votre cours & & 44,8 & 84,6 \\
\hline Courrier électronique (Outlook, Hotmail) & & & 61,5 \\
\hline $\begin{array}{l}\text { Logiciel d'enregistrement audio-vidéo et de capture d'écran } \\
\text { (Panopto, Debut) }\end{array}$ & & & 53,8 \\
\hline DVD & & & 46,2 \\
\hline Forum de discussion & & & 46,2 \\
\hline Outil de recherche bibliographique (Repère, Copernic) & & & 46,2 \\
\hline $\begin{array}{l}\text { Vidéoconférence/Visioconférence en salle ou poste à poste } \\
\text { (Via, Adobe Connect) }\end{array}$ & & & 42,3 \\
\hline
\end{tabular}

Les trois technologies partagées par l'ensemble des profils d'enseignants sont les logiciels de présentation (respectivement, 85,3\%, 92,8 \% et $100 \%$ des petits, moyens et grands utilisateurs), les logiciels de traitement de texte (respectivement, $51,1 \%, 66,3 \%$ et $84,6 \%$ des petits, moyens et grands utilisateurs) et les plateformes d'enseignement (respectivement, 41,3\%, 63\% et 84,6\% des petits, moyens et grands utilisateurs), qui faisaient déjà partie des technologies prégnantes dans la phase de préparation et de gestion de l'enseignement.

Le nombre d'usages mobilisés est plus petit que celui des technologies (voir tableau 3), avec une moyenne de 2, 4 et 7 usages rapportés par les petits, moyens et grands utilisateurs respectivement comme l'illustre le tableau 4. 


\section{Tableau 4}

Pourcentage des usages technologiques les plus fréquemment mobilisés pour le volet de pilotage d'activités d'enseignement en fonction du nombre moyen d'usages technologiques rapporté pour chaque profil d'utilisateur

\begin{tabular}{|l|c|c|c|}
\hline $\begin{array}{l}\text { Classement par ordre décroissant des usages les plus } \\
\text { fréquemment rapportés }\end{array}$ & $\begin{array}{c}\text { Petits utilisateurs } \\
\text { (2 usages en moyenne) }\end{array}$ & $\begin{array}{c}\text { Moyens utilisateurs } \\
\text { (4 usages en } \\
\text { moyenne) }\end{array}$ & $\begin{array}{c}\text { Grands utilisateurs } \\
\text { (7 usages en } \\
\text { moyenne) }\end{array}$ \\
\hline Présenter des documents divers (textes, vidéos, images...) & 77,2 & 91,7 & 92,3 \\
\hline $\begin{array}{l}\text { Communiquer et discuter (échanger avec les étudiants, des } \\
\text { collègues...) }\end{array}$ & 42,4 & 71,8 & 96,2 \\
\hline Supporter les apprentissages des étudiants & & 70,2 & 96,2 \\
\hline Rechercher & & 51,4 & 69,2 \\
\hline Travailler en groupe & & & 76,9 \\
\hline Démontrer les logiciels aux étudiants & & & 73,1 \\
\hline $\begin{array}{l}\text { Prendre des notes ou des informations reliées au contenu de } \\
\text { votre cours }\end{array}$ & & & 69,2 \\
\hline
\end{tabular}

Les usages plus communs entre les profils d'enseignants consistent à présenter des documents (respectivement, $72,2 \%, 91,7 \%$ et $92,3 \%$ des petits, moyens et grands utilisateurs) et à communiquer/discuter (respectivement, $42,4 \%, 71,8 \%$ et $96,2 \%$ des petits, moyens et grands utilisateurs). Ce dernier usage fait de nouveau état d'un différentiel important entre les petits et les grands utilisateurs, ce qui laisse de nouveau penser que la communication est une caractéristique forte des grands utilisateurs.

\section{Développement professionnel}

Finalement, le développement professionnel présente des moyennes de 5, 7 et 14 technologies utilisées respectivement par les petits, moyens et grands utilisateurs comme le laisse voir le tableau 5, ce qui est plus élevé que la phase de pilotage, mais moins élevé que la phase de préparation et de gestion de l'enseignement. 


\section{Tableau 5}

Pourcentage de technologies les plus fréquemment utilisées pour le volet de développement professionnel en fonction du nombre moyen de technologies rapporté pour chaque profil d'utilisateur

\begin{tabular}{|l|c|c|c|}
\hline $\begin{array}{l}\text { Classement par ordre décroissant des technologies les plus } \\
\text { fréquemment rapportées }\end{array}$ & $\begin{array}{l}\text { Petits utilisateurs } \\
\text { (5 tech. utilisées en } \\
\text { moyenne) }\end{array}$ & $\begin{array}{l}\text { Moyens utilisateurs } \\
\text { (7 tech. utilisées en } \\
\text { moyenne) }\end{array}$ & $\begin{array}{l}\text { Grands utilisateurs } \\
\text { (14 tech. utilisées } \\
\text { en moyenne) }\end{array}$ \\
\hline Logiciel de traitement de texte (Word, OpenOffice) & 68,5 & 80,7 & 96,2 \\
\hline Moteur de recherche sur Internet (Google, Yahoo) & 65,2 & 71,3 & 100 \\
\hline Courrier électronique (Outlook, Hotmail) & 64,1 & 74 & 96,2 \\
\hline Logiciel de présentation (PowerPoint, Prezi) & 48,9 & 64,6 & 84,6 \\
\hline Outil de recherche bibliographique (Repère, Copernic) & 35,9 & 49,2 & 76,9 \\
\hline Sites Web reliés au contenu de votre cours & & 56,9 & 88,5 \\
\hline Plateforme d'enseignement (Moodle, Claroline) & & 44,2 & 65,4 \\
\hline Planification de rencontres et sondages (Doodle) & & & 84,6 \\
\hline Logiciel de base de données (Access, SPSS) & & & 65,4 \\
\hline $\begin{array}{l}\text { Vidéoconférence/visioconférence en salle ou poste à poste } \\
\text { (Via, Adobe Connect) }\end{array}$ & & & 61,5 \\
\hline Réseaux sociaux (Twitter, Facebook) & & & 57,7 \\
\hline $\begin{array}{l}\text { Logiciel d'enregistrement audio-vidéo et de capture d'écran } \\
\text { (Panopto, Debut) }\end{array}$ & & & 57,7 \\
\hline Wiki & & & 57,7 \\
\hline Blogues (Blogger, WordPress) & & & 57,7 \\
\hline
\end{tabular}

Les technologies partagées par les trois profils d'enseignants sont le logiciel de traitement de texte (respectivement, $68,5 \%, 80,7 \%$ et $96,2 \%$ des petits, moyens et grands utilisateurs), les moteurs de recherche sur Internet (respectivement, 65,2\%, 71,3\% et $100 \%$ des petits, moyens et grands utilisateurs), le courriel (respectivement, $64,1 \%, 74 \%$ et $96,2 \%$ des petits, moyens et grands utilisateurs), les logiciels de présentation (respectivement, 48,9\%, 64,6\% et 84,6\% des petits, moyens et grands utilisateurs) et les outils de recherche bibliographique (respectivement, $35,9 \%, 49,2 \%$ et $76,9 \%$ des petits, moyens et grands utilisateurs).

Les technologies utilisées pour le développement professionnel servent principalement à rechercher de l'information (respectivement, $82,6 \%, 92,3 \%$ et $100 \%$ des petits, moyens et grands utilisateurs), à accroître ses connaissances (respectivement, 53,3\%,83,4\% et $100 \%$ des petits, moyens et grands utilisateurs) et à consulter, communiquer et partager de l'information (respectivement, $50 \%, 82,3 \%$ et $100 \%$ des petits, moyens et grands utilisateurs), avec une moyenne de 3, 4 et 7 usages mobilisés par les petits, moyens et grands utilisateurs comme cela ressort du tableau 6. 


\section{Tableau 6}

Pourcentage des usages technologiques les plus fréquemment mobilisés pour le volet de développement professionnel en fonction du nombre moyen d'usages technologiques rapporté pour chaque profil d'utilisateur

\begin{tabular}{|l|c|c|c|}
\hline $\begin{array}{l}\text { Classement par ordre décroissant des usages les plus } \\
\text { fréquemment rapportés }\end{array}$ & $\begin{array}{c}\text { Petits utilisateurs } \\
\text { (3 usages en moyenne) }\end{array}$ & $\begin{array}{c}\text { Moyens utilisateurs } \\
\text { (4 usages en } \\
\text { moyenne) }\end{array}$ & $\begin{array}{c}\text { Grands utilisateurs } \\
\text { (7 usages en } \\
\text { moyenne) }\end{array}$ \\
\hline Recherche & 82,6 & 92,3 & 100 \\
\hline Accroissement des connaissances & 53,3 & 83,4 & 100 \\
\hline $\begin{array}{l}\text { Consultation, communication et partage (collègues, sites } \\
\text { d'informations spécialisés) }\end{array}$ & 50 & 82,3 & 100 \\
\hline $\begin{array}{l}\text { Prise de notes ou d'informations pertinentes en lien avec la } \\
\text { profession enseignante }\end{array}$ & & 51,4 & 88,5 \\
\hline Participation à des communautés de pratique & & & 88,5 \\
\hline Meilleure maitrise des outils TIC & & & 80,8 \\
\hline Création de pages Web & & & 53,8 \\
\hline
\end{tabular}

On retrouve ici encore un fort différentiel entre les petits et les grands utilisateurs au sujet des usages communicationnels des technologies, suivi de près par un différentiel lié à l'accroissement des connaissances. Il n'est aucunement possible d'en conclure que les petits utilisateurs communiquent moins ou sont moins enclins à se développer professionnellement. En revanche, il apparaît qu'ils mettent moins à moins profit les technologies à ces fins.

Quelques remarques s'imposent à la vue de ces résultats descriptifs. En premier lieu, les technologies et les usages technologiques sont plus nombreux pour la préparation et la gestion de l'enseignement, suivi du développement professionnel puis du pilotage d'activités. Autrement dit, ils sont davantage mis à profit en dehors de la salle de classe pour soutenir des aspects connexes à l'acte d'enseignement per se. Deuxièmement, les technologies sont systématiquement plus nombreuses que les usages qui en sont faits pour chaque volet d'enseignement. Il apparaît donc que les enseignants utilisent plusieurs technologies pour réaliser des mêmes usages. Troisièmement, les technologies les plus partagées entre les profils d'enseignants semblent se résumer à un nombre relativement réduit «d'incontournables ", à savoir les logiciels de traitement de texte et de présentation, les moteurs de recherche, le courriel et les sites Web reliés aux contenus des cours. À cette base commune initiale viennent se « greffer» d'autres technologies utilisées par les moyens utilisateurs, les grands utilisateurs y greffant à leur tour d'autres technologies. Il en va de même pour les usages, comme le montrent les tableaux 1 à 6 . En somme, les petits, moyens et grands utilisateurs ne se distinguent pas les uns des autres par des technologies et des usages qui seraient spécifiques à leur profil. Ils se démarquent davantage par le fait qu'ils partagent tous un petit lot de technologies et d'usages dont les petits utilisateurs se contentent, alors que les moyens et les grands utilisateurs y ajoutent progressivement d'autres technologies et usages qui accroissent leurs occasions d'intégration pédagogique des technologies. À cet égard, les technologies et les usages technologiques liés à la communication semblent caractériser davantage les grands utilisateurs, ce qui se manifeste par un différentiel plus élevé que les autres technologies et usages technologiques avec les petits utilisateurs. 


\section{Discussion et conclusion}

Pour rappel, les technologies tiennent une place de choix en pédagogie universitaire et ont majoritairement été abordées sous l'angle de leurs effets sur la situation d'enseignement et d'apprentissage en vue d'en révéler l'efficacité pédagogique. Ce faisant, certains autres enjeux, tels que les disparités d'adoption des technologies au sein des enseignants universitaires, sont restés sous-documentés, alors même qu'ils nous apparaissent fondamentaux pour apprécier la contribution réelle des technologies à l'amélioration de la pédagogie universitaire. En nous appuyant sur les constats de Geoghegan (1994) sur les disparités d'adoption des technologies en pédagogie universitaire, l'objectif de cet article était de caractériser les profils d'enseignants universitaires adoptant les technologies. Il ne s'agissait donc pas d'explorer de nouveaux objets de recherche, mais plutôt d'en actualiser certains, pour lesquels il n'existe pas de portrait récent. Pour ce faire, nous avons procédé à une analyse par cluster, qui nous a permis de dégager trois profils d'utilisateurs qui se démarquent significativement par le nombre de technologies et d'usages technologiques mobilisés dans la préparation et la gestion de l'enseignement, le pilotage d'activités et le développement professionnel.

Somme toute, les résultats obtenus aboutissent à un portrait mitigé de l'état d'adoption des technologies par les enseignants universitaires pour deux raisons principales. En premier lieu, les disparités d'adoption des technologies parmi les enseignants universitaires semblent avoir stagné depuis les constats de Geoghegan (1994) puis de Kirkup et al. (2005). Malgré une succession de plus en plus rapide des technologies, les mêmes logiques semblent toujours à l'œuvre, différenciant une petite minorité de grands utilisateurs du reste du corps professoral. À ce titre, les grands utilisateurs sont le profil le moins représentatif des enseignants universitaires, d'abord parce qu'ils ne représentent que $8,7 \%$ de l'échantillon; ensuite, parce qu'ils se démarquent significativement des deux autres profils. D'autre part, bien que tous les participants intègrent les technologies dans leur tâche d'enseignement, ils les mobilisent davantage en dehors des cours (préparation et gestion de l'enseignement; développement professionnel) qu'en salle de classe avec leurs apprenants (pilotage d'activités d'enseignement). Ce résultat est congruent avec d'autres études sur le sujet (Geoghegan, 1994; Usluel et al., 2008). Dans ce contexte, il est peu probable que les technologies contribuent de manière durable et signifiante à l'amélioration de la situation pédagogique, notamment à l'apprentissage des étudiants. Plusieurs auteurs notent à cet effet un décalage entre les attentes et les discours « jovialistes» sur les technologies, comme moteur de transformation, voire de révolution, de la pédagogie universitaire, et leur intégration pédagogique effective (Albero, 2011; Kirkup et al., 2005; Selwyn, 2007). En réaction, d'autres appellent à adopter une posture plus réaliste envers les technologies (Bennett et Maton, 2010; Beynon et al., 1989; Selwyn, 2010).

Il est possible de préciser davantage la nature des disparités observées. En effet, les résultats descriptifs indiquent que les trois profils d'enseignants partagent un petit lot commun de technologies (logiciels de traitement de texte et de présentation, moteurs de recherche, courriel et sites Web reliés aux contenus des cours), dont les petits utilisateurs se contentent alors que les moyens utilisateurs et les grands utilisateurs y ajoutent d'autres technologies et d'autres usages technologiques, ce qui leur confère plus de possibilités d'intégration des technologies à leur enseignement. Ces résultats semblent faire écho à ceux de Fusaro et al. (2012), qui distinguent trois types de technologies rapportées par les enseignants universitaires : les technologies $《$ standards $»^{1}$ (p. ex., courriel, logiciel de traitement de texte et de présentation); les technologies de collaboration et de suivi (p. ex., blogue, wiki); et les technologies spécialisées (p. ex., SPSS). Bien que ces résultats ne soient pas liés à des profils d'enseignants, on retrouve l'idée d'une possible gradation de l'adoption des technologies et des usages technologiques parmi le corps professoral. Ainsi, la différence entre les petits, moyens et grands utilisateurs n'est pas

1 L'emploi des guillemets est le fait des auteurs cités. 
liée aux types de technologies utilisées, puisque les plus populaires sont majoritairement communs, mais à la diversité de technologies auxquelles ils recourent dans leur enseignement. Ce constat rejoint le concept d'échelle d'opportunités (ladder of opportunities) de Livingstone et Helsper (2007) et de Livingstone, Haddon, Görzig et Ólafsson, 2011) à propos des usages d'Internet par les enfants. Partant du fait que le nombre d'usages réalisés sur Internet varie grandement d'un enfant à un autre, ce concept « hypothesises that certain basic activities tend to be done first, and by most children. However, more creative or participatory activities come later, and are undertaken by fewer children » (Livingstone et al., 2011, p. 33). La même logique de différenciation semble s'appliquer aux enseignants universitaires.

Comment expliquer cette relative stagnation de l'état d'adoption des technologies et des disparités qui en résultent? La réponse semble en partie due au fait que les plans institutionnels d'intégration des technologies sont pensés et mis en œuvre à partir de l'archétype des grands utilisateurs, négligeant de fait les besoins spécifiques des autres profils d'enseignants universitaires, qui sont pourtant majoritaires. À l'instar de Kirkup et al. (2005), il nous semble important de ne pas « extrapolate from the actions and enthusiasm of early adopters in order to predict the use and impact on the larger scale » (p. 2). Aussi, les initiatives institutionnelles mises en œuvre pour favoriser l'intégration pédagogique des technologies devraient davantage cibler les moyens et petits utilisateurs en veillant à s'adapter à leurs caractéristiques spécifiques (voir Geoghegan, 1994).

Cette étude, dont les résultats aboutissent à une caractérisation plutôt cohérente des profils d'enseignants universitaires, n'est toutefois pas sans limites, lesquelles pourraient utilement orienter des pistes de recherche futures. La première d'entre elles a trait au fait que l'échantillon n'est pas représentatif, non seulement parce qu'il s'agit d'un échantillon de convenance, mais surtout parce que l'invitation à répondre au questionnaire s'est déroulée par courriel, ce qui a tendance à privilégier les utilisateurs fréquents des technologies (Hargittai, 2010). Dans cette perspective, les non-utilisateurs sont d'emblée exclus et les petits utilisateurs sont généralement sous-représentés. En outre, l'étude se base sur les perceptions des participants plutôt que sur leur utilisation effective des technologies. De plus, les variables utilisées pour mesurer l'adoption des technologies, bien qu'elles soient fiables, restent partielles et mériteraient d'être enrichies pour représenter plus finement les disparités entre enseignants. Enfin, les résultats, bien qu'ils proviennent de deux universités volontairement contrastées, n'ont pas de force prédictive et ne peuvent donc être généralisés au-delà de cette étude, bien que rien ne permette de penser qu'ils soient substantiellement différents dans les autres universités du Québec. Une piste de recherche future consisterait à répliquer l'étude à plus grande échelle en enrichissant les variables mesurant l'adoption des technologies et en recourant à des méthodes de collecte additionnelles, telles que les observations in situ. Sachant que les moyens et petits utilisateurs sont souvent négligés dans les politiques d'intégration des technologies, une autre piste de recherche consisterait à documenter plus finement leurs besoins en vue d'orienter les stratégies institutionnelles vers une meilleure prise en compte de ces profils majoritaires d'enseignants.

\section{Références}

Albero, B. (2011). Le couplage entre pédagogie et technologies à l'université : cultures d'action et paradigmes de recherche. Revue internationale des technologies en pédagogie universitaire, 8(1-2), 11-21. doi:10.7202/1005779ar

Baron, G.-L. et Bruillard, É. (1996). L’informatique et ses usagers dans l'éducation. Paris : Presses universitaires de France.

Bennett, S. et Maton, K. (2010). Beyond the "digital natives" debate: Towards a more nuanced understanding of students' technology experiences. Journal of Computer Assisted Learning, 26(5), 321-331. doi:10.1111/j.13652729.2010.00360.x 
Ben Youssef, A., Ben Youssef, H. et Dahmani, M. (2013). Higher education teachers e-skills and the innovation process. International Journal of Computer and Information Technology, 2(2), 185-195. Récupéré de l'archive ouverte HAL : http://halshs.archives-ouvertes.fr

Beynon, W. M., Norris, M. T., Russ, S. B., Slade, M. D., Yung, Y. P. et Yung, Y. W. (1989). Software construction using definitions: an illustrative example. Récupéré de Warwick Research Archive Portal : http://wrap.warwick.ac.u

Collin, S. et Karsenti, T. (2013). Approche théorique des usages des technologies en education: regard critique. Formation et profession: revue internationale en éducation, 20(3), 89-101.

Cuban, L. (1986). Teachers and machines: The classroom use of technology since 1920. New York, NY : Teachers College Press.

De Ketele, J.-M. (2010). La pédagogie universitaire : un courant en plein développement. Revue française de pédagogie, (172), 5-13. Récupéré de http://rfp.revues.org

De Tarde, G. (1890). Les lois de l'imitation : étude sociologique. Paris, France : Félix Alcan.

Edmunds, R., Thorpe, M. et Conole, G. (2012). Student attitudes towards and use of ICT in course study, work and social activity: A technology acceptance model approach. British Journal of Educational Technology, 43(1), 71-84. doi:10.1111/j.1467-8535.2010.01142.x

Fusaro, M., Couture, A., Venkatesh, V., Rocheleau, J., Larose, M. et Chassé, D. (2012). Études sur les modalités d'apprentissage et les technologies de l'information et de la communication dans l'enseignement (rapport du Groupe de travail sur l'étude des usages des technologies de l'information et de la communication dans l'enseignement). Récupéré du site du Bureau de coopération internationale : http://www.crepuq.qc.ca

Gall, J. P., Gall, M. D. et Borg, W. R. (2005). Applying educational research: A practical guide ( $5^{\mathrm{e}}$ éd.). Boston, MA : Pearson Education.

Geoghegan, W. H. (1994). What ever happened to instructional technology?. Récupéré de http://eprints.soton.ac.uk

Gul, I., Arif, M. et Yousaf, M. J. (2013). Adoption and diffusion of information and communication technologies in higher education: using structural equation modelling. Dans M. Ahmad (dir.), Proc. 10th Int. Conference on Statistical Sciences (p. 133-148). Récupéré du site de Islamic Countries Society of Statistical Sciences :

http://www.isoss.net

Hargittai, E. (2010). Digital na(t)ives? Variation in internet skills and uses among members of the "net generation". Sociological inquiry, 80(1), 92-113. doi:10.1111/j.1475682x.2009.00317.x
Helsper, E. J. et Eynon, R. (2010). Digital natives: Where is the evidence? British Educational Research Journal, 36(3), 503-520. doi:10.1080/01411920902989227

Karsenti, T., Raby, C., Villeneuve, S. et Gauthier, C. (2007). La formation des maîtres et la manifestation de la compétence professionnelle à intégrer les technologies de l'information et des communications (TIC) aux fins de préparation et de pilotage d'activités d'enseignement-apprentissage, de gestion de l'enseignement et de développement professionnel (rapport détaillé de recherche). Récupéré de http://depot.erudit.org/

Kerr, S. T. (1996). Toward a sociology of educational technology. Dans D. H. Jonassen et M. Driscoll (dir.), Handbook of research on educational communications and technology: A project of the Association for educational communications and technology ( $2^{\mathrm{e}}$ éd., p. 37-59). Londres, R.-U. : Lawrence Erlbaum.

Kirkup, C., Sizmur, J., Sturman, L. et Lewis, K. (2005). Schools' use of data in teaching and learning (rapport de recherche $\left.\mathrm{n}^{\circ} \mathrm{RR} 671\right)$. Récupéré des archives du gouvernement du Royaume-Uni : http://webarchive.nationalarchives.gov.uk

Larose, F., Lenoir, Y., Karsenti, T. et Grenon, V. (2002). Les facteurs sous-jacents au transfert des compétences informatiques construites par les futurs maîtres du primaire sur le plan de l'intervention éducative. Revue des sciences de l'éducation, 28(2), 265-287. doi:10.7202/007354ar

Livingstone, S. et Helsper, E. (2007). Gradations in digital inclusion: Children, young people and the digital divide. New media \& society, 9(4), 671-696. doi:10.1177/14614 44807080335

Livingstone, S., Haddon, L., Görzig, A. et Ólafsson, K. (2011). Risks and safety on the Internet: The perspective of European children. Récupéré du site du London School of Economics and Political Science : http://www.lse.ac.uk

Millerand, F. (1998). Usages des NTIC : les approches de la diffusion, de l'innovation et de l'appropriation ( $1^{\text {ère }}$ partie). Commposite, 2(1), 1-19. Récupéré de http://www.commposite.org

Ministère de l'Éducation du Québec. (2001). La formation à l'enseignement professionnel : les orientations, les compétences professionnelles. Récupéré de http://www.education.gouv.qc.ca

Moore, G. A. (1991). Crossing the chasm: Marketing and selling technology products to mainstream customers. New York, NY : Harper Business.

Raby, C., Boegner-Pagé, S., Charron, A., Gagnon, B. et Bouchard, A.-P. (2013). Le développement de la compétence professionnelle des enseignants du préscolaire et du primaire à intégrer les TIC en classe : impact d'une recherche-action. Formation et profession : revue scientifique internationale en éducation, 21(2), 19-33. doi:10.18162/fp.2013.2 
Rogers, E. M. (2003). The diffusion of innovations (5éd.). New York, NY : The Free Press.

Selwyn, N. (2007). The use of computer technology in university teaching and learning: a critical perspective. Journal of Computer Assisted Learning, 23(2), 83-94. doi:10.1111/j.1365-2729.2006.00204.x

Selwyn, N. (2010). Looking beyond learning: Notes towards the critical study of educational technology. Journal of Computer Assisted Learning, 26(1), 65-73. doi:10.1111/ j.1365-2729.2009.00338.x

Selwyn, N. (2015). Technology and education - why it's crucial to be critical. Dans S. Bulfin, N. F. Johnson et C. Bigum (dir.), Critical perspectives on technology and education (p. 245-255). New York, NY : Palgrave Macmillan. doi:10.1057/9781137385451_14

Stockless, A. et Beaupré, J. (2014). Résultats du sondage technopédagogique auprès des enseignants du primaire et du secondaire. Récupéré de http://infogr.am/

Usluel, Y. K., Aşkar, P. et Baş, T. (2008). A structural equation model for ICT usage in higher education. Journal of Educational Technology \& Society, 11(2), 262-273. Récupéré de http://www.ifets.info

Villeneuve, S., Karsenti, T., Raby, C. et Meunier, H. (2011). Les futurs enseignants du Québec sont-ils technocompétents? Une analyse en fonction de la compétence professionnelle à intégrer les TIC. Revue internationale des technologies en pédagogie universitaire, 9(1-2), 78-99. doi:10.7202/1012904ar

Wei, L. (2012). Number matters: The multimodality of Internet use as an indicator of the digital inequalities. Journal of Computer-Mediated Communication, 17(3), 303-318. doi:10.1111/j.1083-6101.2012.01578.x 